SLAC-PUB-10576

July 2004

\title{
Conformal Symmetry as a Template for QCD*
}

\author{
Stanley J. Brodsky \\ Stanford Linear Accelerator Center \\ Stanford University, Stanford, California 94309 \\ E-mail: sjbth@slac.stanford.edu
}

\begin{abstract}
Conformal symmetry is broken in physical QCD; nevertheless, one can use conformal symmetry as a template, systematically correcting for its nonzero $\beta$ function as well as higher-twist effects. For example, commensurate scale relations which relate QCD observables to each other, such as the generalized Crewther relation, have no renormalization scale or scheme ambiguity and retain a convergent perturbative structure which reflects the underlying conformal symmetry of the classical theory. The "conformal correspondence principle" also dictates the form of the expansion basis for hadronic distribution amplitudes. The AdS/CFT correspondence connecting superstring theory to superconformal gauge theory has important implications for hadron phenomenology in the conformal limit, including an all-orders demonstration of counting rules for hard exclusive processes as well as determining essential aspects of hadronic light-front wavefunctions. Theoretical and phenomenological evidence is now accumulating that QCD couplings based on physical observables such as $\tau$ decay become constant at small virtuality; i.e., effective charges develop an infrared fixed point in contradiction to the usual assumption of singular growth in the infrared. The near-constant behavior of effective couplings also suggests that QCD can be approximated as a conformal theory even at relatively small momentum transfer. The importance of using an analytic effective charge such as the pinch scheme for unifying the electroweak and strong couplings and forces is also emphasized.
\end{abstract}

Invited talk given at the

Workshop Continuous Advances in QCD 2004

William I. Fine Theoretical Physics Institute

University of Minnesota, Minneapolis

May 13-16, 2004

\footnotetext{
*Work supported by Department of Energy contract DE-AC03-76SF00515.
} 


\section{Introduction: The Conformal Correspondence Principle}

The classical Lagrangian of QCD for massless quarks is conformally symmetric. Since it has no intrinsic mass scale, the classical theory is invariant under the $S O(4,2)$ translations, boosts, and rotations of the Poincare group, plus the dilatations and other transformations of the conformal group. Scale invariance and therefore conformal symmetry is destroyed in the quantum theory by the renormalization procedure which introduces a renormalization scale as well as by quark masses. Conversely, Parisi [1] has shown that perturbative QCD becomes a conformal theory for $\beta \rightarrow 0$ and zero quark mass. Conformal symmetry is thus broken in physical QCD; nevertheless, we can still recover the underlying features of the conformally invariant theory by evaluating any expression in QCD in the analytic limit of zero quark mass and zero $\beta$ function:

$$
\lim _{m_{q} \rightarrow 0, \beta \rightarrow 0} \mathcal{O}_{Q C D}=\mathcal{O}_{\text {conformal QCD }}
$$

This conformal correspondence limit is analogous to Bohr's correspondence principle where one recovers predictions of classical theory from quantum theory in the limit of zero Planck constant. The contributions to an expression in QCD from its nonzero $\beta$-function can be systematically identified [2, 3, 4] order-by-order in perturbation theory using the Banks-Zaks procedure [5].

There are a number of useful phenomenological consequences of near conformal behavior of QCD: the conformal approximation with zero $\beta$ function can be used as template for QCD analyses [6, 7] such as the form of the expansion polynomials for distribution amplitudes [8, 9]. The near-conformal behavior of QCD is the basis for commensurate scale relations [10] which relate observables to each other without renormalization scale or scheme ambiguities [2, 3. By definition, all contributions from the nonzero $\beta$ function can be incorporated into the QCD running coupling $\alpha_{s}(Q)$ where $Q$ represents the set of physical invariants. Conformal symmetry thus provides a template for physical QCD expressions. For example, perturbative expansions in QCD for massless quarks must have the form

$$
\mathcal{O}=\sum_{n=0} C_{n} \alpha_{s}^{n}\left(Q_{n}^{*}\right)
$$

where the $C_{n}$ are identical to the expansion coefficients in the conformal theory, and $Q_{n}^{*}$ is the scale chosen to resum all of the contributions from the nonzero $\beta$ function at that order in perturbation theory. Since the conformal theory does not contain renormalons, the $C_{n}$ do not have the divergent $n$ ! growth characteristic of conventional PQCD expansions evaluated at a fixed scale. 


\section{Effective Charges}

One can define the fundamental coupling of QCD from virtually any physical observable [1]. Such couplings, called "effective charges", are all-order resummations of perturbation theory, so they correspond to the complete theory of QCD. Unlike the $\overline{\mathrm{MS}}$ coupling, a physical coupling is analytic across quark flavor thresholds [12, 13. In particular, heavy particles will contribute to physical predictions even at energies below their threshold. This is in contrast to mathematical renormalization schemes such as $\bar{M} S$, where mass thresholds are treated as step functions. In addition, since the QCD running couplings defined from observables are bounded, integrations over effective charges are well defined and the arguments requiring renormalon resummations do apply. The physical couplings satisfy the standard renormalization group equation for its logarithmic derivative, $\mathrm{d} \alpha_{\text {phys }} / \mathrm{d} \ln k^{2}=\widehat{\beta}_{\text {phys }}\left[\alpha_{\text {phys }}\left(k^{2}\right)\right]$, where the first two terms in the perturbative expansion of $\widehat{\beta}_{\text {phys }}$ are scheme-independent at leading twist; the higher order terms have to be calculated for each observable separately using perturbation theory.

Commensurate scale relations are QCD predictions which relate observables to each other at their respective scales. An important example is the generalized Crewther relation [14]:

$$
\left[1+\frac{\alpha_{R}\left(s^{*}\right)}{\pi}\right]\left[1-\frac{\alpha_{g_{1}}\left(Q^{2}\right)}{\pi}\right]=1
$$

where the underlying form at zero $\beta$ function is dictated by conformal symmetry [15]. Here $\alpha_{R}(s) / \pi$ and $-\alpha_{g_{1}}\left(Q^{2}\right) / \pi$ represent the entire radiative corrections to $R_{e^{+} e^{-}}(s)$ and the Bjorken sum rule for the $g_{1}\left(x, Q^{2}\right)$ structure function measured in spindependent deep inelastic scattering, respectively. The relation between $s^{*}$ and $Q^{2}$ can be computed order by order in perturbation theory, as in the BLM method [16]. The ratio of physical scales guarantees that the effect of new quark thresholds is commensurate. Commensurate scale relations are renormalization-scheme independent and satisfy the group properties of the renormalization group. Each observable can be computed in any convenient renormalization scheme such as dimensional regularization. The $\overline{\mathrm{MS}}$ coupling can then be eliminated; it becomes only an intermediary 10]. In such a procedure there are no further renormalization scale $(\mu)$ or scheme ambiguities.

In the case of QED, the heavy lepton potential (in the limit of vanishing external charge) is conventionally used to define the effective charge $\alpha_{q e d}\left(q^{2}\right)$. This definition, the Dyson Goldberger-Low effective charge, resums all lepton pair vacuum polarization contributions in the photon propagator, and it is analytic in the lepton masses. The scale of the QCD coupling is thus the virtuality of the exchanged photon. The extension of this concept to non-abelian gauge theories is non-trivial due to the self interactions of the gauge bosons which make the usual self-energy gauge dependent. However, by systematically implementing the Ward identities of the theory, one can project out the unique self-energy of each physical particle. This results in a glu- 
onic self-energy which is gauge independent and which can be resummed to define an effective charge that is related through the optical theorem to differential cross sections. The algorithm for performing the calculation at the diagrammatic level is called the "pinch technique" [17, 18, 19, 20]. The generalization of the pinch technique to higher loops has recently been investigated [21, 22, 23, 24, 25, 26]. Binosi and Papavassiliou 24, 25, 26] have shown the consistency of the pinch technique to all orders in perturbation theory, thus allowing a systematic application to the QCD and electroweak effective charges at higher orders. The pinch scheme is in fact used to define the evolution of the couplings in the electroweak theory. The pinch scheme thus provides an ideal scheme for QCD couplings as well.

\section{Effective Charges and Unification}

Recently Michael Binger and I have analyzed a supersymmetric grand unification model in the context of physical renormalization schemes [27. Our essential assumption is that the underlying forces of the theory become at the unification scale. We have found a number of qualitative differences and improvements in precision over conventional approaches. There is no need to assume that the particle spectrum has any specific structure; the effect of heavy particles is included both below and below the physical threshold. Unlike mathematical schemes such as dimensional reduction, $\overline{D R}$, the evolution of the coupling is analytic and unification is approached continuously rather than at a fixed scale. The effective charge formalism thus provides a template for calculating all mass threshold effects for any given grand unified theory. These new threshold corrections are important in making the measured values of the gauge couplings consistent with unification. A comparison with the conventional scheme based on $\overline{D R}$ dimensional regularization scheme is summarized in Fig. 1,

\section{The Infrared Behavior of Effective QCD Cou- plings}

It is often assumed that color confinement in QCD can be traced to the singular behavior of the running coupling in the infrared, i.e. "infrared slavery." For example if $\alpha_{s}\left(q^{2}\right) \rightarrow 1 / q^{2}$ at $q^{2} \rightarrow 0$, then one-gluon exchange leads to a linear potential at large distances. However, theoretical [28, 29, 30, 31, 32] and phenomenological [33, 34, 35] evidence is now accumulating that the QCD coupling becomes constant at small virtuality; i.e., $\alpha_{s}\left(Q^{2}\right)$ develops an infrared fixed point in contradiction to the usual assumption of singular growth in the infrared. Since all observables are related by commensurate scale relations, they all should have an IR fixed point 31]. A recent study of the QCD coupling using lattice gauge theory in Landau gauge in fact shows an

infrared fixed point [36]. This result is also consistent with Dyson-Schwinger equation 


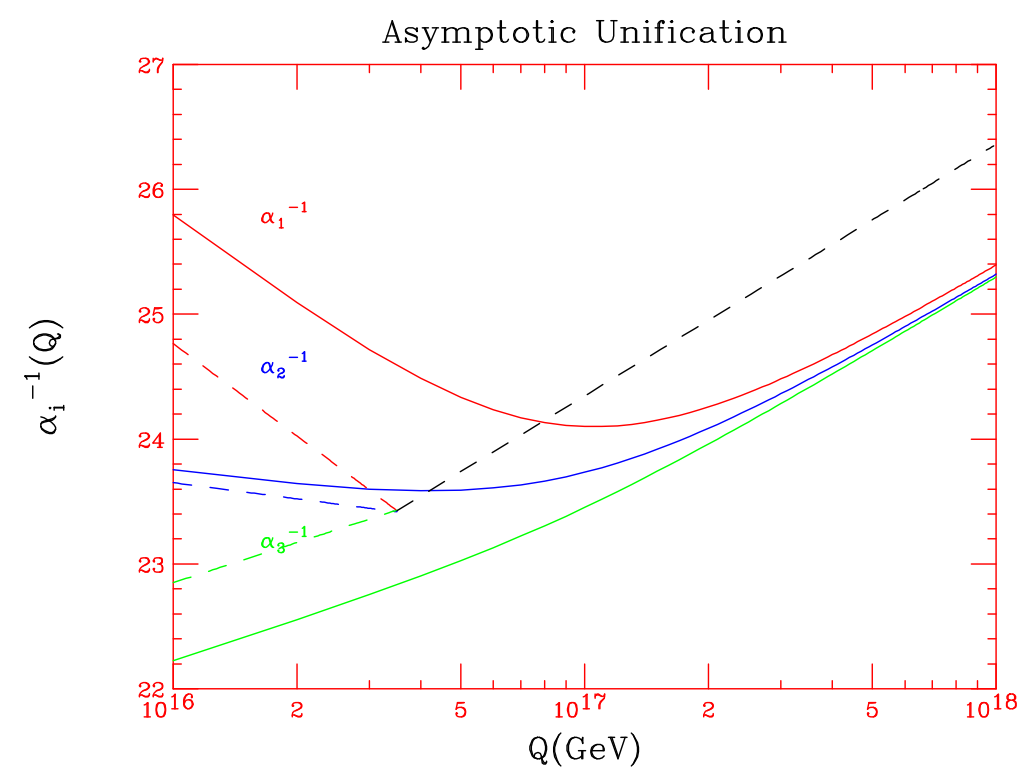

Figure 1: Asymptotic Unification. An illustration of strong and electroweak coupling unification in an $S U(5)$ supersymmetric model based on the pinch scheme effective charge. The solid lines are the analytic pinch scheme $\overline{P T}$ effective couplings, while the dashed lines are the $\overline{D R}$ couplings. For illustrative purposes, $a_{3}\left(M_{Z}\right)$ has been chosen so that unification occurs at a finite scale for $\overline{D R}$ and asymptotically for the $\overline{P T}$ couplings. Here $M_{S U S Y}=200 \mathrm{GeV}$ is the mass of all light superpartners except the wino and gluino which have values $\frac{1}{2} m g x=M_{S U S Y}=2 m_{w x}$.

studies of the physical gluon propagator [28, 29]. The relationship of these results to the infrared-finite coupling for the vector interaction defined in the quarkonium potential has recently been discussed by Badalian and Veselov [37.

Menke, Merino, and Rathsman [34] and I have considered a physical coupling for QCD which is defined from the high precision measurements of the hadronic decay channels of the $\tau^{-} \rightarrow \nu_{\tau} \mathrm{h}^{-}$. Let $R_{\tau}$ be the ratio of the hadronic decay rate to the leptonic rate. Then $R_{\tau} \equiv R_{\tau}^{0}\left[1+\frac{\alpha_{\tau}}{\pi}\right]$, where $R_{\tau}^{0}$ is the zeroth order QCD prediction, defines the effective charge $\alpha_{\tau}$. The data for $\tau$ decays is well-understood channel by channel, thus allowing the calculation of the hadronic decay rate and the effective charge as a function of the $\tau$ mass below the physical mass. The vector and axialvector decay modes which can be studied separately. Using an analysis of the $\tau$ data from the OPAL collaboration [38, we have found that the experimental value of the coupling $\alpha_{\tau}(s)=0.621 \pm 0.008$ at $s=m_{\tau}^{2}$ corresponds to a value of $\alpha_{\overline{\mathrm{MS}}}\left(M_{Z}^{2}\right)=(0.117$ $0.122) \pm 0.002$, where the range corresponds to three different perturbative methods used in analyzing the data. This result is in good agreement with the world average $\alpha_{\overline{\mathrm{MS}}}\left(M_{Z}^{2}\right)=0.117 \pm 0.002$. However, from the figure we also see that the effective charge only reaches $\alpha_{\tau}(s) \sim 0.9 \pm 0.1$ at $s=1 \mathrm{GeV}^{2}$, and it even stays within the same range down to $s \sim 0.5 \mathrm{GeV}^{2}$. This result is in good agreement with the 
estimate of Mattingly and Stevenson [33] for the effective coupling $\alpha_{R}(s) \sim 0.85$ for $\sqrt{s}<0.3 \mathrm{GeV}$ determined from $\mathrm{e}^{+} \mathrm{e}^{-}$annihilation, especially if one takes into account the perturbative commensurate scale relation, $\alpha_{\tau}\left(m_{\tau^{\prime}}^{2}\right)=\alpha_{R}\left(s^{*}\right)$, where $s^{*} \simeq 0.10 m_{\tau^{\prime}}^{2}$. This behavior is not consistent with the coupling having a Landau pole, but rather shows that the physical coupling is close to constant at low scales, suggesting that physical QCD couplings are effectively constant or "frozen" at low scales.

Figure 2 shows a comparison of the experimentally determined effective charge $\alpha_{\tau}(s)$ with solutions to the evolution equation for $\alpha_{\tau}$ at two-, three-, and four-loop order normalized at $m_{\tau}$. At three loops the behavior of the perturbative solution drastically changes, and instead of diverging, it freezes to a value $\alpha_{\tau} \simeq 2$ in the infrared. The infrared behavior is not perturbatively stable since the evolution of the coupling is governed by the highest order term. This is illustrated by the widely different results obtained for three different values of the unknown four loop term $\beta_{\tau, 3}$ which are also shown. The values of $\beta_{\tau, 3}$ used are obtained from the estimate of the four loop term in the perturbative series of $R_{\tau}, K_{4}^{\overline{\mathrm{MS}}}=25 \pm 50$ [39]. It is interesting to note that the central four-loop solution is in good agreement with the data all the way down to $s \simeq 1 \mathrm{GeV}^{2}$.

The results for $\alpha_{\tau}$ resemble the behavior of the one-loop "time-like" effective coupling 40, 41, 42,

$$
\alpha_{\text {eff }}(s)=\frac{4 \pi}{\beta_{0}}\left\{\frac{1}{2}-\frac{1}{\pi} \arctan \left[\frac{1}{\pi} \ln \frac{s}{\Lambda^{2}}\right]\right\}
$$

which is finite in the infrared and freezes to the value $\alpha_{\text {eff }}(s)=4 \pi / \beta_{0}$ as $s \rightarrow 0$. It is instructive to expand the "time-like" effective coupling for large $s$,

$$
\begin{aligned}
\alpha_{\mathrm{eff}}(s) & =\frac{4 \pi}{\beta_{0} \ln \left(s / \Lambda^{2}\right)}\left\{1-\frac{1}{3} \frac{\pi^{2}}{\ln ^{2}\left(s / \Lambda^{2}\right)}+\frac{1}{5} \frac{\pi^{4}}{\ln ^{4}\left(s / \Lambda^{2}\right)}+\ldots\right\} \\
& =\alpha_{\mathrm{s}}(s)\left\{1-\frac{\pi^{2} \beta_{0}^{2}}{3}\left(\frac{\alpha_{\mathrm{s}}(s)}{4 \pi}\right)^{2}+\frac{\pi^{4} \beta_{0}^{4}}{5}\left(\frac{\alpha_{\mathrm{s}}(s)}{4 \pi}\right)^{4}+\ldots\right\} .
\end{aligned}
$$

This shows that the "time-like" effective coupling is a resummation of $\left(\pi^{2} \beta_{0}^{2} \alpha_{\mathrm{s}}^{2}\right)^{n_{-}}$ corrections to the usual running couplings. The finite coupling $\alpha_{\text {eff }}$ given in Eq. (4) obeys standard PQCD evolution at LO. Thus one can have a solution for the perturbative running of the QCD coupling which obeys asymptotic freedom but does not have a Landau singularity.

The near constancy of the effective QCD coupling at small scales illustrates the near-conformal behavior of QCD. It helps explain the empirical success of dimensional counting rules for the power law fall-off of form factors and fixed angle scaling. As shown in the references 43, 44, one can calculate the hard scattering amplitude $T_{H}$ for such processes [45] without scale ambiguity in terms of the effective charge $\alpha_{\tau}$ or $\alpha_{R}$ using commensurate scale relations. The effective coupling is evaluated in the regime where the coupling is approximately constant, in contrast to the rapidly 


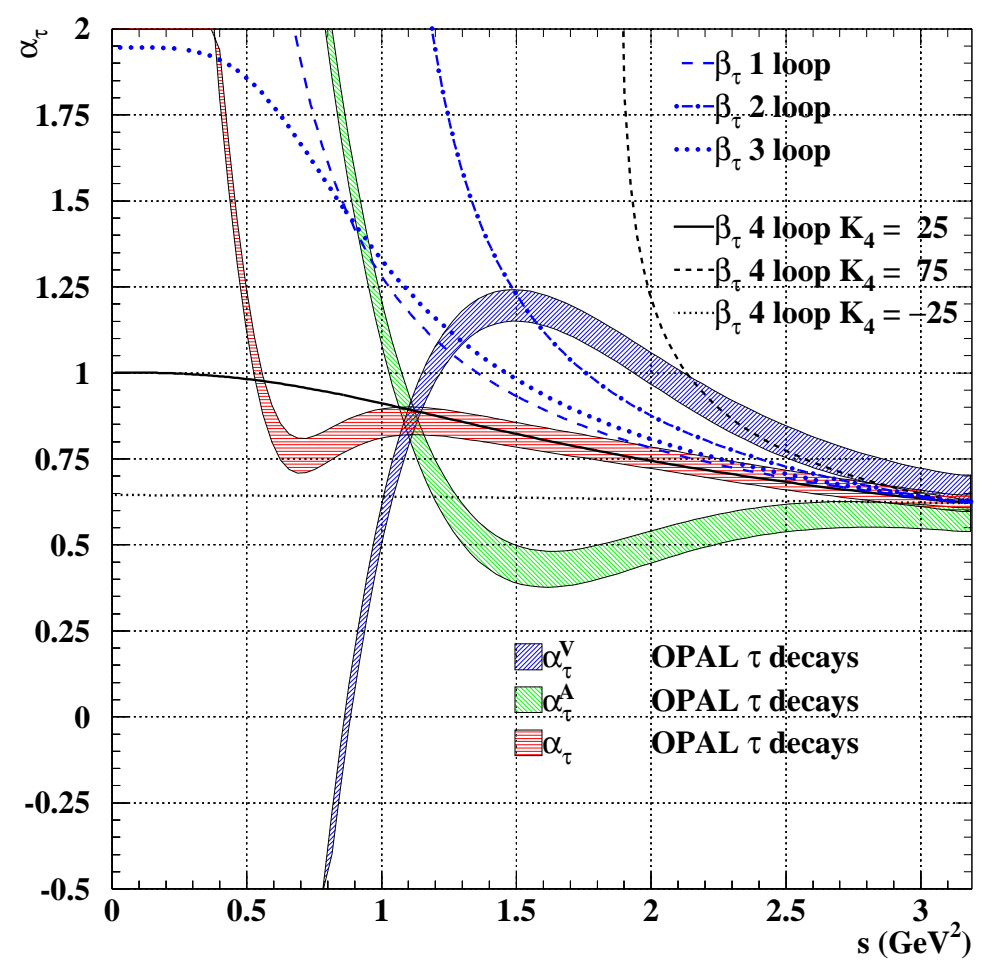

Figure 2: The effective charge $\alpha_{\tau}$ for non-strange hadronic decays of a hypothetical $\tau$ lepton with $m_{\tau^{\prime}}^{2}=s$ compared to solutions of the fixed order evolution equation for $\alpha_{\tau}$ at two-, three-, and four-loop order. The error bands include statistical and systematic errors.

varying behavior from powers of $\alpha_{\mathrm{s}}$ predicted by perturbation theory (the universal two-loop coupling). For example, the nucleon form factors are proportional at leading order to two powers of $\alpha_{\mathrm{s}}$ evaluated at low scales in addition to two powers of $1 / q^{2}$; The pion photoproduction amplitude at fixed angles is proportional at leading order to three powers of the QCD coupling. The essential variation from leading-twist counting-rule behavior then only arises from the anomalous dimensions of the hadron distribution amplitudes.

\section{Light-Front Quantization}

The concept of a wave function of a hadron as a composite of relativistic quarks and gluons is naturally formulated in terms of the light-front Fock expansion at fixed light-front time, $\tau=x \cdot \omega$. The four-vector $\omega$, with $\omega^{2}=0$, determines the orientation of the light-front plane; the freedom to choose $\omega$ provides an explicitly covariant 
formulation of light-front quantization [46]. Although LFWFs depend on the choice of the light-front quantization direction, all observables such as matrix elements of local current operators, form factors, and cross sections are light-front invariants they must be independent of $\omega_{\mu}$.

The light-front wave functions (LFWFs) $\psi_{n}\left(x_{i}, k_{\perp_{i}}, \lambda_{i}\right)$, with $x_{i}=\frac{k_{i} \cdot \omega}{P \cdot \omega}, \sum_{i=1}^{n} x_{i}=$ $1, \sum_{i=1}^{n} k_{\perp_{i}}=0_{\perp}$, are the coefficient functions for $n$ partons in the Fock expansion, providing a general frame-independent representation of the hadron state. Matrix elements of local operators such as spacelike proton form factors can be computed simply from the overlap integrals of light front wave functions in analogy to nonrelativistic Schrödinger theory. In principle, one can solve for the LFWFs directly from fundamental theory using nonperturbative methods such as discretized lightfront quantization (DLCQ), the transverse lattice, lattice gauge theory moments, or Bethe-Salpeter techniques. The determination of the hadron LFWFs from phenomenological constraints and from QCD itself is a central goal of hadron and nuclear physics. Reviews of nonperturbative light-front methods may be found in the references [47, 46, 48, 49. A potentially important method is to construct the $q \bar{q}$ Green's function using light-front Hamiltonian theory, with DLCQ boundary conditions and Lippmann-Schwinger resummation. The zeros of the resulting resolvent projected on states of specific angular momentum $J_{z}$ can then generate the meson spectrum and their light-front Fock wavefunctions. The DLCQ properties and boundary conditions allow a truncation of the Fock space while retaining the kinematic boost and Lorentz invariance of light-front quantization.

One of the central issues in the analysis of fundamental hadron structure is the presence of non-zero orbital angular momentum in the bound-state wave functions. The evidence for a "spin crisis" in the Ellis-Jaffe sum rule signals a significant orbital contribution in the proton wave function [50, 51]. The Pauli form factor of nucleons is computed from the overlap of LFWFs differing by one unit of orbital angular momentum $\Delta L_{z}= \pm 1$. Thus the fact that the anomalous moment of the proton is non-zero requires nonzero orbital angular momentum in the proton wavefunction [52]. In the light-front method, orbital angular momentum is treated explicitly; it includes the orbital contributions induced by relativistic effects, such as the spin-orbit effects normally associated with the conventional Dirac spinors.

In recent work, Dae Sung Hwang, John Hiller, Volodya Karmonov [53], and I have studied the analytic structure of LFWFs using the explicitly Lorentz-invariant formulation of the front form. Eigensolutions of the Bethe-Salpeter equation have specific angular momentum as specified by the Pauli-Lubanski vector. The corresponding LFWF for an $n$-particle Fock state evaluated at equal light-front time $\tau=\omega \cdot x$ can be obtained by integrating the Bethe-Salpeter solutions over the corresponding relative light-front energies. The resulting LFWFs $\psi_{n}^{I}\left(x_{i}, k_{\perp i}\right)$ are functions of the light-cone momentum fractions $x_{i}=k_{i} \cdot \omega / p \cdot \omega$ and the invariant mass squared of the constituents $M_{0}^{2}=\left(\sum_{i=1}^{n} k_{i}^{\mu}\right)^{2}=\sum_{i=1}^{n}\left[\frac{k_{\perp}^{2}+m^{2}}{x}\right]_{i}$ and the light-cone momentum fractions $x_{i}=k \cdot \omega / p \cdot \omega$ each multiplying spin-vector and polarization tensor invari- 
ants which can involve $\omega^{\mu}$. The resulting LFWFs for bound states are eigenstates of the Karmanov-Smirnov kinematic angular momentum operator [54] and satisfy all of the Lorentz symmetries of the front form, including boost invariance.

\section{AFS/CFT Correspondence and Hadronic Light- Front Wavefunctions}

As shown by Maldacena [55], there is a remarkable correspondence between large $N_{C}$ supergravity theory in a higher dimensional anti-de Sitter space and supersymmetric QCD in 4-dimensional space-time. String/gauge duality provides a framework for predicting QCD phenomena based on the conformal properties of the AdS/CFT correspondence. The AdS/CFT correspondence is based on the fact that the generators of conformal and Poincare transformations have representations on the five-dimensional anti-deSitter space $A d S_{5}$ as well as Minkowski spacetime. For example, Polchinski and Strassler [56] have shown that the power-law fall-off of hard exclusive hadronhadron scattering amplitudes at large momentum transfer can be derived without the use of perturbation theory by using the scaling properties of the hadronic interpolating fields in the large- $r$ region of AdS space. Thus one can use the Maldacena correspondence to compute the leading power-law falloff of exclusive processes such as high-energy fixed-angle scattering of gluonium-gluonium scattering in supersymmetric QCD. The resulting predictions for hadron physics effectively coincide [56, 57, 58] with QCD dimensional counting rules: [59, 60, 61]

$$
\frac{d \sigma}{d t}\left(H_{1} H_{2} \rightarrow H_{3} H_{4}\right)=\frac{F(t / s)}{s^{n-2}}
$$

where $n$ is the sum of the minimal number of interpolating fields in the initial and final state. (For a recent review of hard fixed $\theta_{C M}$ angle exclusive processes in QCD see reference [62.) As shown by Brower and Tan [57], the non-conformal dimensional scale which appears in the QCD analysis is set by the string constant, the slope of the primary Regge trajectory $\Lambda^{2}=\alpha_{R}^{\prime}(0)$ of the supergravity theory. Polchinski and Strassler [56] have also derived counting rules for deep inelastic structure functions at $x \rightarrow 1$ in agreement with perturbative QCD predictions 63] as well as Bloom-Gilman exclusive-inclusive duality.

The supergravity analysis is based on an extension of classical gravity theory in higher dimensions and is nonperturbative. Thus analyses of exclusive processes [45] which were based on perturbation theory can be extended by the Maldacena correspondence to all orders. An interesting point is that the hard scattering amplitudes which are normally or order $\alpha_{s}^{p}$ in PQCD appear as order $\alpha_{s}^{p / 2}$ in the supergravity predictions. This can be understood as an all-orders resummation of the effective potential [55, 64].

The superstring theory results are derived in the limit of a large $N_{C}$ [65]. For

gluon-gluon scattering, the amplitude scales as $1 / N_{C}^{2}$. For color-singlet bound states 


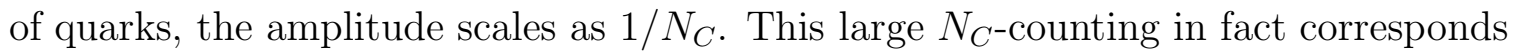
to the quark interchange mechanism 66]. For example, for $K^{+} p \rightarrow K^{+} p$ scattering, the $u$-quark exchange amplitude scales approximately as $\frac{1}{u} \frac{1}{t^{2}}$, which agrees remarkably well with the measured large $\theta_{C M}$ dependence of the $K^{+} p$ differential cross section [67]. This implies that the nonsinglet Reggeon trajectory asymptotes to a negative integer [68, in this case, $\lim _{-t \rightarrow \infty} \alpha_{R}(t) \rightarrow-1$.

De Teramond and I 69 have shown how to compute the form and scaling of light-front hadronic wavefunctions using the AdS/CFT correspondence in quantum field theories which have an underlying conformal structure, such as $\mathcal{N}=4$ superconformal QCD. For example, baryons are included in the theory by adding an open string sector in $A d S_{5} \times S^{5}$ corresponding to quarks in the fundamental representation of the $S U(4)$ symmetry defined on $S^{5}$ and the fundamental and higher representations of $S U\left(N_{C}\right)$. The hadron mass scale is introduced by imposing boundary conditions at the $A d S_{5}$ coordinate $r=r_{0}=\Lambda_{Q C D} R^{2}$. The quantum numbers of the lowest Fock state of each hadron including its internal orbital angular momentum and spin-flavor symmetry, are identified by matching the fall-off of the string wavefunction $\Psi(x, r)$ at the asymptotic $3+1$ boundary. Higher Fock states are identified with conformally invariant quantum fluctuations of the bulk geometry about the AdS background. The scaling and conformal properties of the AdS/CFT correspondence leads to a hard component of the LFWFs of the form:

$$
\begin{aligned}
\psi_{n / h}\left(x_{i}, \vec{k}_{\perp i}, \lambda_{i}, l_{z i}\right) & \sim \frac{\left(g_{s} N_{C}\right)^{\frac{1}{2}(n-1)}}{\sqrt{N_{C}}} \prod_{i=1}^{n-1}\left(k_{i \perp}^{ \pm}\right)^{\left|l_{z i}\right|} \\
& \times\left[\frac{\Lambda_{o}}{M^{2}-\sum_{i} \frac{\vec{k}_{\perp i}^{2}+m_{i}^{2}}{x_{i}}+\Lambda_{o}^{2}}\right]^{n+\left|l_{z}\right|-1},
\end{aligned}
$$

where $g_{s}$ is the string scale and $\Lambda_{o}$ represents the basic QCD mass scale. The scaling predictions agree with perturbative QCD analyses [70, 45], but the AdS/CFT analysis is performed at strong coupling without the use of perturbation theory. The nearconformal scaling properties of light-front wavefunctions lead to a number of other predictions for QCD which are normally discussed in the context of perturbation theory, such as constituent counting scaling laws for the leading power fall-off of form factors and hard exclusive scattering amplitudes for QCD processes. The ratio of Pauli to Dirac baryon form factor have the nominal asymptotic form $F_{2}\left(Q^{2}\right) / F_{1}\left(Q^{2}\right) \sim$ $1 / Q^{2}$, modulo logarithmic corrections, in agreement with the perturbative results [71]. Our analysis can also be extended to study the spin structure of scattering amplitudes at large transverse momentum and other processes which are dependent on the scaling and orbital angular momentum structure of light-front wavefunctions. 


\section{Acknowledgements}

This talk is based on collaborations with Michael Binger, Gregory Gabadadze, Einan Gardi, Georges Grunberg, John Hiller, Dae Sung Hwang, Volodya Karmanov, Andre Kataev, Hung Jung Lu, Sven Menke, Carlos Merino, Johan Rathsman, and Guy de Téramond. I am also thankful to the William I. Fine Theoretical Physics Institute at the University of Minnesota which sponsored this meeting. This work was supported by the U.S. Department of Energy, contract DE-AC03-76SF00515.

\section{References}

[1] G. Parisi, Phys. Lett. B 39, 643 (1972).

[2] S. J. Brodsky, E. Gardi, G. Grunberg and J. Rathsman, Phys. Rev. D 63, 094017 (2001) arXiv:hep-ph/0002065.

[3] J. Rathsman, in Proc. of the 5th International Symposium on Radiative Corrections (RADCOR 2000) ed. Howard E. Haber, arXiv:hep-ph/0101248.

[4] G. Grunberg, JHEP 0108, 019 (2001) arXiv:hep-ph/0104098.

[5] T. Banks and A. Zaks, Nucl. Phys. B 196, 189 (1982).

[6] S. J. Brodsky, Y. Frishman and G. P. Lepage, Phys. Lett. B 167, 347 (1986).

[7] S. J. Brodsky, P. Damgaard, Y. Frishman and G. P. Lepage, Phys. Rev. D 33, 1881 (1986).

[8] V. M. Braun, G. P. Korchemsky and D. Muller, Prog. Part. Nucl. Phys. 51, 311 (2003) arXiv:hep-ph/0306057.

[9] V. M. Braun, S. E. Derkachov, G. P. Korchemsky and A. N. Manashov, Nucl. Phys. B 553, 355 (1999) arXiv:hep-ph/9902375.

[10] S. J. Brodsky and H. J. Lu, Phys. Rev. D 51, 3652 (1995) arXiv:hep-ph/9405218.

[11] G. Grunberg, Phys. Lett. B95, 70 (1980) [Erratum-ibid. B110, 501 (1982)]. G. Grunberg, Phys. Rev. D29, 2315 (1984).

[12] S. J. Brodsky, M. S. Gill, M. Melles and J. Rathsman, Phys. Rev. D 58, 116006 (1998) arXiv:hep-ph/9801330.

[13] S. J. Brodsky, M. Melles and J. Rathsman, Phys. Rev. D 60, 096006 (1999) arXiv:hep-ph/9906324. 
[14] S. J. Brodsky, G. T. Gabadadze, A. L. Kataev and H. J. Lu, Phys. Lett. B372, 133 (1996) arXiv:hep-ph/9512367.

[15] R. J. Crewther, Phys. Rev. Lett. 28, 1421 (1972).

[16] S. J. Brodsky, G. P. Lepage and P. B. Mackenzie, Phys. Rev. D 28, 228 (1983).

[17] J. M. Cornwall, Phys. Rev. D 26, 1453 (1982).

[18] G. Degrassi and A. Sirlin, Phys. Rev. D 46, 3104 (1992).

[19] N.J. Watson. Nucl. Phys. B 494, 388-432 (1997).

[20] J. Papavassiliou, E. de Rafael, N.J. Watson. Nucl. Phys. B 503, 79 (1997). hep-ph/9612237.

[21] N. J. Watson, Nucl. Phys. Proc. Suppl. 74, 341 (1999) arXiv:hep-ph/9812203.

[22] N.J. Watson. Nucl. Phys. B 552, 461 (1999). hep-ph/9812202.

[23] J. Papavassiliou. Phys. Rev. Lett. 84, 2782 (2000). hep-ph/9912336.

[24] D. Binosi and J. Papavassiliou, Phys. Rev. D 66, 111901 (2002). hep-ph/0208189.

[25] D. Binosi and J. Papavassiliou, Nucl. Phys. Proc. Suppl. 121, 281 (2003). hep-ph/0209016.

[26] D. Binosi, arXiv:hep-ph/0401182.

[27] M. Binger and S. J. Brodsky, Phys. Rev. D 69, 095007 (2004) arXiv:hep-ph/0310322.

[28] L. von Smekal, R. Alkofer and A. Hauck, Phys. Rev. Lett. 79, 3591 (1997) arXiv:hep-ph/9705242.

[29] D. Zwanziger, Phys. Rev. D 69, 016002 (2004) arXiv:hep-ph/0303028.

[30] D. M. Howe and C. J. Maxwell, Phys. Lett. B 541, 129 (2002) arXiv:hep-ph/0204036.

[31] D. M. Howe and C. J. Maxwell, Phys. Rev. D 70, 014002 (2004) arXiv:hep-ph/0303163.

[32] S. Furui and H. Nakajima, arXiv:hep-lat/0309166.

[33] A. C. Mattingly and P. M. Stevenson, Phys. Rev. D 49, 437 (1994) arXiv:hep-ph/9307266. 
[34] S. J. Brodsky, S. Menke, C. Merino and J. Rathsman, Phys. Rev. D 67, 055008 (2003) arXiv:hep-ph/0212078.

[35] M. Baldicchi and G. M. Prosperi, Phys. Rev. D 66, 074008 (2002) arXiv:hep-ph/0202172.

[36] S. Furui and H. Nakajima, arXiv:hep-lat/0403021.

[37] A. M. Badalian and A. I. Veselov, arXiv:hep-ph/0407082.

[38] K. Ackerstaff et al. [OPAL Collaboration], Eur. Phys. J. C 7, 571 (1999) arXiv:hep-ex/9808019.

[39] F. Le Diberder and A. Pich, Phys. Lett. B289, 165 (1992).

[40] M. Beneke and V. M. Braun, Phys. Lett. B348, 513 (1995) arXiv:hep-ph/9411229.

[41] P. Ball, M. Beneke and V. M. Braun, Nucl. Phys. B452, 563 (1995) arXiv:hep-ph/9502300.

[42] Y. L. Dokshitzer, G. Marchesini and B. R. Webber, Nucl. Phys. B469, 93 (1996) arXiv:hep-ph/9512336.

[43] S. J. Brodsky, C. R. Ji, A. Pang and D. G. Robertson, Phys. Rev. D 57, 245 (1998) arXiv:hep-ph/9705221.

[44] B. Melic, B. Nizic and K. Passek, Phys. Rev. D 65, 053020 (2002) arXiv:hep-ph/0107295.

[45] G. P. Lepage and S. J. Brodsky, Phys. Rev. D 22, 2157 (1980).

[46] J. Carbonell, B. Desplanques, V. A. Karmanov, and J. F. Mathiot, Phys. Rep. 300, 215 (1998) arXiv:nucl-th/9804029.

[47] S. J. Brodsky, H. C. Pauli and S. S. Pinsky, Phys. Rept. 301, 299 (1998) arXiv:hep-ph/9705477.

[48] S. Dalley, Nucl. Phys. B (Proc. Suppl.) 108, 145 (2002).

[49] S. J. Brodsky, Published in Nagoya 2002, Strong coupling gauge theories and effective field theories, 1-18. arXiv:hep-th/0304106.

[50] R. L. Jaffe and A. Manohar, Nucl. Phys. B 337, 509 (1990).

[51] X. D. Ji, Nucl. Phys. Proc. Suppl. 119, 41 (2003) arXiv:hep-lat/0211016.

[52] S. J. Brodsky and S. D. Drell, Phys. Rev. D 22, 2236 (1980). 
[53] S. J. Brodsky, J. R. Hiller, D. S. Hwang and V. A. Karmanov, Phys. Rev. D 69, 076001 (2004) arXiv:hep-ph/0311218.

[54] V. A. Karmanov and A.V. Smirnov, Nucl. Phys. A 546, 691 (1992).

[55] J. M. Maldacena, Adv. Theor. Math. Phys. 2, 231 (1998) [Int. J. Theor. Phys. 38, 1113 (1999)] arXiv:hep-th/9711200.

[56] J. Polchinski and M. J. Strassler, Phys. Rev. Lett. 88, 031601 (2002) arXiv:hep-th/0109174.

[57] R. C. Brower and C. I. Tan, Nucl. Phys. B 662, 393 (2003) arXiv:hep-th/0207144.

[58] O. Andreev, Phys. Rev. D 67, 046001 (2003) arXiv:hep-th/0209256.

[59] S. J. Brodsky and G. R. Farrar, Phys. Rev. Lett. 31, 1153 (1973).

[60] V. A. Matveev, R. M. Muradian and A. N. Tavkhelidze, Lett. Nuovo Cim. 7, 719 (1973).

[61] S. J. Brodsky and G. R. Farrar, Phys. Rev. D 11, 1309 (1975).

[62] S. J. Brodsky, Published in Newport News 2002, Exclusive processes at high momentum transfer 1-33. arXiv:hep-ph/0208158.]

[63] S. J. Brodsky, M. Burkardt and I. Schmidt, Nucl. Phys. B 441, 197 (1995) arXiv:hep-ph/9401328.

[64] S. J. Rey and J. T. Yee, Eur. Phys. J. C 22, 379 (2001) arXiv:hep-th/9803001.

[65] G. 't Hooft, Nucl. Phys. B 72, 461 (1974).

[66] J. F. Gunion, S. J. Brodsky and R. Blankenbecler, Phys. Rev. D 8, 287 (1973).

[67] D. W. Sivers, S. J. Brodsky and R. Blankenbecler, Phys. Rept. 23, 1 (1976).

[68] R. Blankenbecler et al., Phys. Rev. D 8, 4117 (1973).

[69] S. J. Brodsky and G. F. de Teramond, Phys. Lett. B 582, 211 (2004) arXiv:hep-th/0310227.

[70] X. D. Ji, F. Yuan and J. P. Ma, Phys. Rev. Lett. 90, 241601 (2003).

[71] A. V. Belitsky, X. D. Ji and F. Yuan, Phys. Rev. Lett. 91, 092003 (2003) arXiv:hep-ph/0212351. 